\title{
Classical and molecular methods in differentiation of mantle cell lymphoma and small lymphocytic lymphoma in composite lymphoma: a case report
}

\author{
Michał Szymczyk ${ }^{1}$ - Grzegorz Rymkiewicz ${ }^{2}$ - Zbigniew Bystydzieński ${ }^{2}$ - Małgorzata Szostakowska-Rodzoś ${ }^{3}$. \\ Renata Zub $^{3} \cdot$ Renata Woroniecka ${ }^{4} \cdot$ Ewa Paszkiewicz-Kozik $^{1} \cdot$ Anna Fabisiewicz $^{3}$ (I)
}

Received: 20 July 2020 / Accepted: 12 November 2020 / Published online: 7 January 2021

(C) Springer-Verlag GmbH Germany, part of Springer Nature 2021

\begin{abstract}
Composite lymphoma (CL) is a very rare phenomenon. To date, only few cases of composite mantle cell lymphoma (MCL) and chronic lymphocytic leukemia (CLL) or small lymphocytic lymphoma (SLL) have been reported by Sun et al. (Cytometry B Clin Cytom 2018;94(1):148-50), Hoeller et al. (Hum Pathol 2013;44(1):110-21), and Papathomas et al. (Hum Pathol 2012;43(4):46780). In the majority of CL cases, the clonality relationship was not examined or could not be proven. So far, there is no optimal treatment strategy for CL. However, it has been a commonly accepted rule that the more aggressive component should be treated first. Thus, it is of vital importance to precisely distinguish the components of CL and to determine the response of a particular component to treatment. This may be done by monitoring the molecular minimal residual disease (mMRD) as reported by Pott et al. (Blood 2010;115(16):3215-23) and Pott (Semin Hematol 2011;48(3):172-84). This report presents the possibility of determining clonality of each CL component by using flow cytometry in parallel with specific molecular markers. Molecular analysis was performed by quantitative real-time PCR (qPCR) using two markers: $I G H$ rearrangement as reported by van Dongen et al. (Leukemia 2003;17(12):2257-317) and SOX11 expression by Hamborg et al. (Eur J Haematol 2012;89(5):38594) and Szostakowska et al. (Med Oncol 2018;35(4):49). The SOX11 expression allowed us to distinguish MCL and SLL subclones in a 60 -year-old male with multifocal MCL/SLL as this marker is not detected in SLL as reported by Swerdlow et al. (2001) and Mozos et al. (Haematologica 2009;94(11):1555-62). To our best knowledge, this is the first example of the use of mMRD to distinguish CL components.
\end{abstract}

Keywords Composite lymphoma $\cdot$ Mantle cell lymphoma $\cdot$ Small lymphocytic lymphoma $\cdot$ Flow cytometry $\cdot$ Minimal residual disease · Quantitative real-time PCR

Anna Fabisiewicz

anna.fabisiewicz@pib-nio.pl

1 Department of Lymphoid Malignancies, Maria Skłodowska-Curie National Research Institute of Oncology, Roentgena 5, 02-781 Warsaw, Poland

2 Flow Cytometry Laboratory, Department of Pathology and Laboratory Diagnostics, Maria Skłodowska-Curie National Research Institute of Oncology, Roentgena 5, 02-781 Warsaw, Poland

3 Department of Molecular and Translational Oncology, Maria Skłodowska-Curie National Research Institute of Oncology, Roentgena 5, 02-781 Warsaw, Poland

4 Cytogenetic Laboratory, Maria Skłodowska-Curie National Research Institute of Oncology, Roentgena 5, 02-781 Warsaw, Poland

\section{Introduction}

Composite lymphoma (CL) is defined as the coexistence of two different types of lymphoma in the same organ or tissue site [1]. It can be composed of either non-Hodgkin lymphoma (NHL) (B cell or T cell) or Hodgkin lymphoma. Here, we describe a case of composite mantle cell lymphoma (MCL) and small lymphocytic lymphoma (SLL).

MCL is a rare (5-8\% of all NHL) and incurable B-NHL characterized by a multiple relapsing and aggressive course in most cases. The hallmark of the disease is a translocation $\mathrm{t}(11 ; 14)(\mathrm{q} 13 ; \mathrm{q} 32)$ resulting in overexpression of cyclin D1 [2]. In rare cyclin D1 negative cases, the presence of SOX11 may allow the correct final diagnosis [3]. The MCL immunophenotype is common to the B lymphocytes: CD19+/ CD20+/CD22+/cyclin D1+/CD200- with coexpression of 
CD5+/CD43+ (characteristic for the T cell linage) and sIgM+/ $\operatorname{sIgD} \pm$ with $\lambda>\mathrm{k}$ monoclonal restriction. Malignant cells are usually negative for CD23, CD11c, CD10, and BCL6 [2] although there are some CD5-, CD10+, and CD23+ cases (e.g., splenic type). The disease usually presents as a generalized lymphadenopathy, with common bone marrow (BM), peripheral blood (PB), and gastrointestinal tract involvement. There is no defined therapeutic standard; however, the goal of the first line therapy is to achieve complete remission [4], most preferable at a molecular level assessed by qPCR analysis [5-8].

CLL and SLL are the most common types of adult leukemia/lymphoma which account for approximately $30 \%$ of all NHL [9]. According to the 2016 World Health Organization (WHO) lymphoma classification, although CLL and SLL are the same disease, the term SLL is used for cases with a circulating SLL cell count $<5 \times 10^{9} / \mathrm{L}$ and documented nodal, splenic, or other extramedullary involvement. Lymph nodes are infiltrated by small CD19+/CD20+ ${ }^{\mathrm{dim}} /$ CD22-/CD23+ ${ }^{\mathrm{dim}} /$ cyclin D1-/CD200+ with coexpression of CD5+/CD43+ and sIgM+/sIgD \pm B lymphocytes [10-12]. SLL/CLL has a highly variable clinical course: from aggressive forms (with a complex karyotype and TP53 mutations/ deletions) which should be treated at diagnosis to indolent forms that do not require treatment for many years and require only a watch-and-wait approach [13].

Relapse due to undetermined minimal residual disease (MRD) is the main cause of death in lymphoma patients; therefore, monitoring of MRD is crucial in the process of treatment. Recent studies have shown that consistent monitoring of MRD can improve treatment decisions [6]. Moreover, in patients who are in clinical remission, MRD status estimated in PB was prognostic for their overall survival (OS) [14]. Therefore, consistent monitoring of MRD could allow improvements to current treatments and predict clinical relapse, progressive-free survival (PFS), and OS. In this clinical case, we aimed to use mMRD assessment as an indication for deciding on treatment specific for relapsing subclone of the CL.

\section{Clinical history}

Here, we report a case of a 60-year-old male with multifocal MCL/SLL composite lymphoma who presented with unspecified pain in the lower part of the abdomen, periodic diarrheas and rectal hemorrhages, night sweats, and weight loss over a period of 6 months. The patient was also diagnosed with diabetes type 2, persistent atrial fibrillation, and arterial hypertension. He had no other medical history. In the local hospital, he was diagnosed for colon polyposis or inflammatory bowel disease. The colonoscopy revealed multifocal flat polyps. The histopathology examination indicated the possibility of MCL presented as lymphomatoid polyposis. The patient was transferred to the Department of Lymphoid Malignancies at the Maria Sklodowska-Curie National Research Institute of
Oncology, Warsaw, Poland. Physical examination revealed numerous enlarged superficial lymph nodes (armpits, groins, neck, supraclavicular, and submandibular). He had no palpable organomegaly. The flow cytometry (FCM), classical cytogenetics (CC), and fluorescence in situ hybridization (FISH) examinations of fine needle aspiration biopsy (FNAB) of an enlarged $3-\mathrm{cm}$ axillary lymph node (LN) and BM/PB revealed MCL as well as SLL. To confirm the diagnosis, a right neck lymphadenectomy was performed. We received 4 swollen and enlarged LNs, each of them approximately $2 \mathrm{~cm}$ long.

The diagnosis results are shown in Table 1 and Figs. 1 and 2. Histopathology and immunohistochemistry of this CL case are shown in Fig. 1. Cervical LN biopsy showed a coexistence of SLL and MCL. Both clones expressed CD20, and the SLL component was $\mathrm{CD} 23+$, cyclin D1, and SOX11 negative, while the MCL component was CD23-, cyclin D1, and SOX11 positive. The Ki-67 index was about 5\% for SLL and about $40 \%$ for MCL. Detailed results are summarized in Table 1.

Figure 2 shows flow cytometric immunophenotypic analysis of cells obtained by FNAB from an enlarged lymph node. Analysis of the expression of selected antigens shows the coexistence of two separate neoplastic clones originating from B cells. Cells belonging to both subpopulations, marked with the R1 gate in the scattergram A, are not different from each other in terms of size (forward scatter, FSC) and granulation (side scatter, SSC).

The phenotype of the MCL subpopulation demonstrates expression of surface antigens most commonly encountered in routine FCM diagnostics as previously described [2]. Considering differences in its median fluorescence intensity (MFI) levels, this phenotype may be characterized by having the following: high CD20 expression of bright type, reduced CD19 expression in comparison to normal B cells, medium CD5/CD45 coexpression (weaker than that of T cells), CD23 -, high CD79b expression, low CD25 coexpression, CD200-, medium-high CD38 expression, low IgD expression, medium-high IgM expression, and CD71 expression in nearly $50 \%$ of cells.

Unlike in MCL, the phenotype of the coexisting SLL subpopulation is different from that in the typical form of this disease. The coexisting SLL phenotype has bright, medium CD20 expression (in the classic forms, CD20 expression is weak and dim), low peak IgD expression, and lacks IgM expression (in typical SLL, the accumulation of both chains is most commonly of low intensity). The absence of CD25 coexpression which is relatively uncommon in SLL is also observed. Moreover, there is no CD38 expression, characteristic of the nonmutated SLL form associated with a less favorable prognosis. Furthermore, CD200+, CD5+, CD23+, $\mathrm{CD} 19+, \mathrm{CD} 79 \mathrm{~b} \pm$, and CD71+ occur in a low percentage of cells. This immunophenotype clearly indicated the diagnosis of SLL as previously described [11]. 


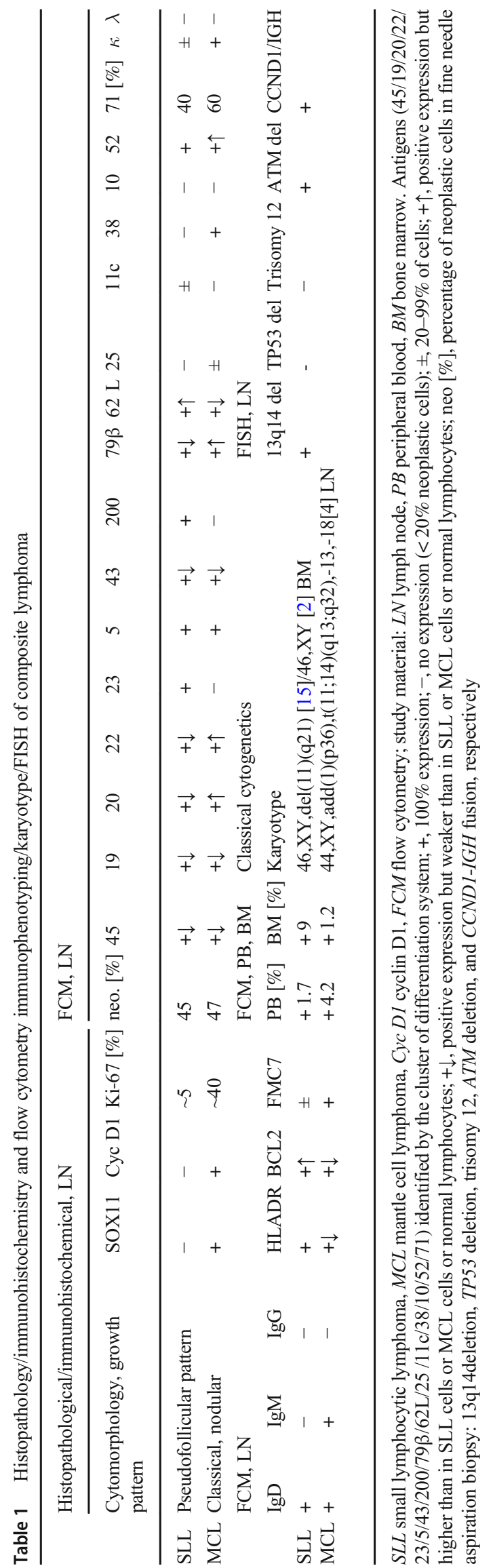

The karyotype demonstrated the presence of two different clones: one with 11q deletion in BM and the second with a $\mathrm{t}(11 ; 14)(\mathrm{q} 13 ; \mathrm{q} 32)$ in LN (Table 1). However, FISH performed on LN confirmed both above aberrations in neoplastic cells: ATM (11q22.3) deletion and CCND1-IGH, observed in 15\% and $23 \%$ of cells, respectively. Moreover, FISH revealed $13 \mathrm{q} 14$ deletion in $83 \%$ of cells. CC and FISH results confirmed occurrence of two subpopulations: SLL with ATM deletion and MCL with CCND1-IGH, both carrying 13q14 deletion. Standard PB examinations revealed grade 2 [according to the Common Terminology Criteria for Adverse Events (CTCAE) ] anemia and a mild hyperleukocytosis with an elevated lymphocyte count. The lactate dehydrogenase (LDH) was elevated (276 IU/L, upper normal range $227 \mathrm{IU} / \mathrm{L})$. The baseline $\mathrm{PB}$ examination revealed $6.73 \mathrm{G} / \mathrm{L}$ WBC with a pathological MCL population at a level of about 280 cells in $1 \mathrm{~mm}^{3}$ of PB $(4.1 \%$ of PB nucleated elements and $0.28 \mathrm{G} / \mathrm{L})$ and SLL subclone at a level of about 115 cells in $1 \mathrm{~mm}^{3}$ of PB $(1.7 \%$ of nucleated blood cells and $0.11 \mathrm{G} / \mathrm{L})$.

The patient was in good general condition [grade 1 according to Eastern Cooperative Oncology Group (ECOG) performance status scale]. Whole body computed tomography (CT) revealed numerous enlarged lymph nodes in the cervix, the armpit, and near the stomach as well as enlarged mesenteric, external, and inguinal lymph nodes. Massive infiltration of the stomach wall, duodenum, jejunum, ileum, caecum, and rectum as well as an enlarged spleen was also visible in CT. MCL was diagnosed as stage IVB according to Ann Arbor classification with a high Mantle Cell International Prognostic Index (MIPI) score predicting a shorter and more aggressive disease course. SLL was classified as stage IVB. Due to bulky disease, the patient started treatment with fractionated doses of RCHOP (rituximab, cyclophosphamide, adriamycin, vincristine, and prednisone). The patient received 6 courses of alternating RCHOP and RIVAC (rituximab, ifosfamide, etoposide, and cytarabine) immunochemotherapy. After the fourth course of induction (second RIVAC), two successful aphaereses were performed. The midterm evaluation revealed partial remission according to the Cheson response criteria. The patient then became eligible for autologous hematopoietic cell transplantation (auto-HCT). Unexpectedly, just before the auto-HCT, the disease progression of SLL was detected. The colonoscopy revealed massive infiltration of the large intestine by SLL. The FCM of PB/BM confirmed SLL progression without features of Richter syndrome transformation [11] and MCL partial remission. The patient died due to SLL rapid progression.

\section{Molecular analysis}

Gene Scan molecular analysis of IGH amplified sequences was performed on $\mathrm{PB}$ and $\mathrm{BM}$ samples at the time of diagnosis. This examination demonstrated two peaks: $341 \mathrm{bp}$ and 
Fig. 1 Histopathology and immunohistochemistry of composite lymphoma. a Cervical lymph node biopsy showing the coexistence of small lymphocytic lymphoma (SLL) (red stamp in the lower right corner) and mantle cell lymphoma (MCL) (green stamp in the upper left corner). Diffuse growth of SLL small lymphocytes and a slightly larger monotonous population of medium-sized centrocytic cells with scant cytoplasm. Scattered "pink" macrophages in MCL component (green arrows in the upper left corner (hematoxylin and eosin)). b-d SLL (on the right) and MCL (on the left): CD20 expression stronger in MCL component than in SLL; CD23 positive in SLL but negative in MCL and cyclin D1 negative in SLL comparing to positive in MCL. Original magnification $\times 100$

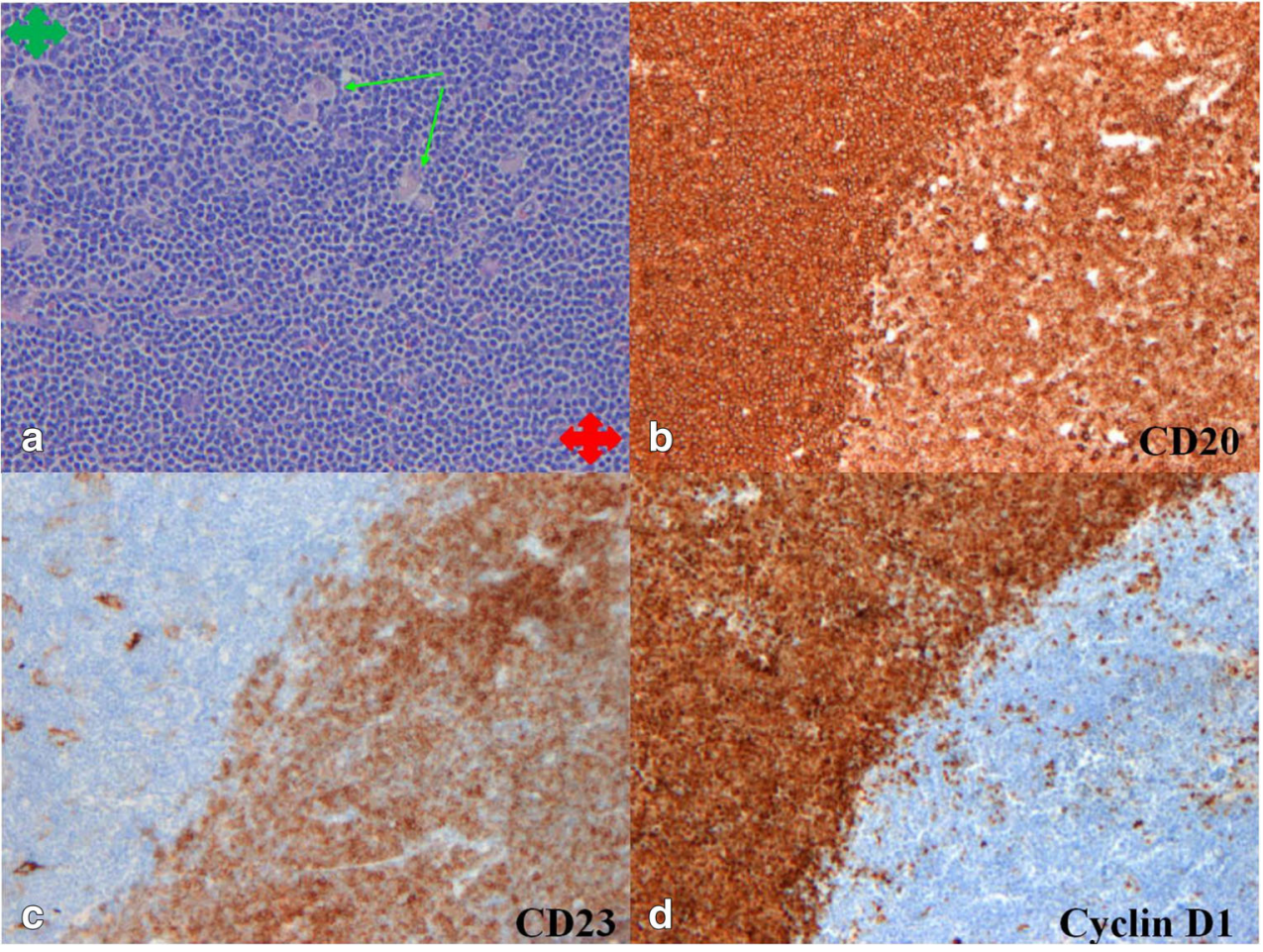

$349 \mathrm{bp}$, confirming the presence of two different clonal rearrangements: VH3, JH4 and VH4, JH6 which indicated lack of a clonality relationship.

Sequence analysis using an IMGT/V-QUEST tool (www. imgt.org) of junctional regions [13] showed that one of the clones had developed by recombination of IGHVH3-21*01F, $\mathrm{D} 3-9 * 01 \mathrm{~F}$, and $\mathrm{JH} 4 * 02 \mathrm{~F}$ regions while the second one by recombination of IGHVH4-59*01F, D3-22*01F, and $\mathrm{JH} 6 * 02 \mathrm{~F}$.

\begin{tabular}{lllll}
\hline $\begin{array}{l}341 \text { bp peak } \\
\text { tgtgcgag }\end{array}$ & gaggcc & attacgatatttgactggttattat & gaa & actactttgactactgg \\
$3^{\prime} \mathrm{VH} 3$ & $\mathrm{~N} 1$ & $\mathrm{D} 3$ region & $\mathrm{N} 2$ & $5^{\prime} \mathrm{JH} 4$ region \\
349 bp peak & & & ctcc & actactactacggtatggacgtctgg \\
tgtgcgagag & ctcgtgcgg & attactatgatagtagtggttat & $\mathrm{N} 2$ & $5^{\prime} \mathrm{JH} 6$ region \\
$3^{\prime} \mathrm{VH} 4$ & $\mathrm{~N} 1$ & $\mathrm{D} 3$ region &
\end{tabular}

\section{Monitoring of mMRD during follow-up}

Apart from midterm clinical evaluation, an analysis of the level of mMRD was performed in order to determine the effectiveness of chemotherapy. Molecular markers have greater sensitivity than the FCM [6]; therefore, in this study, they were chosen as the standard method for MRD monitoring. For this purpose, in follow-up samples, an $I G H$ rearrangement with 2 different starter sets was analyzed by qPCR [7] and SOX11 expression was estimated. The SOX11 marker is not expressed in SLL [16]; therefore, it is detectable only in MCL. The regression analysis for the V3J4 and V4J6 rearrangements with $S O X 11$ expression showed a relationship between
V4J6 and SOX11 $\left(R^{2}=0.9966\right)$ indicating that this $I G H$ rearrangement is a characteristic of MCL (Fig. 3). Based on this analysis, we considered the V4J6 rearrangement specific for MCL.

The MRD was also set by standard FCM, using the starting FCM immunophenotypic features of both CL components, at three time points: at the time of diagnosis, during treatment, and at the end of the treatment. The MRD assessment during the patient's treatment showed decreasing levels of MCL and SLL. In one PB sample taken in the middle of the treatment, the qPCR analysis showed an increased level of SLL (Fig. 4, top, left). This unexpected finding is difficult to explain when looking at the other results from that timepoint which 
Fig. 2 Fine needle aspiration biopsy/flow cytometry analysis of composite lymphoma.

Coexistence of small lymphocytic lymphoma (SLL) cells (red), mantle cell lymphoma (MCL) cells (green), normal B cells (black dots in boxes), and $\mathrm{T}$ lymphocytes. a Forward scatter (FSC)/side scatter (SSC) scattergram showing only one cluster of cells in terms of size in $\mathrm{R} 1$ region. $\mathbf{b}$ and $\mathbf{d}-\mathbf{I}$ Scattergrams showing the different patterns of expression of analyzed markers in SLL and MCL. c Showing the same CD5/CD19 expression on SLL and MCL cells
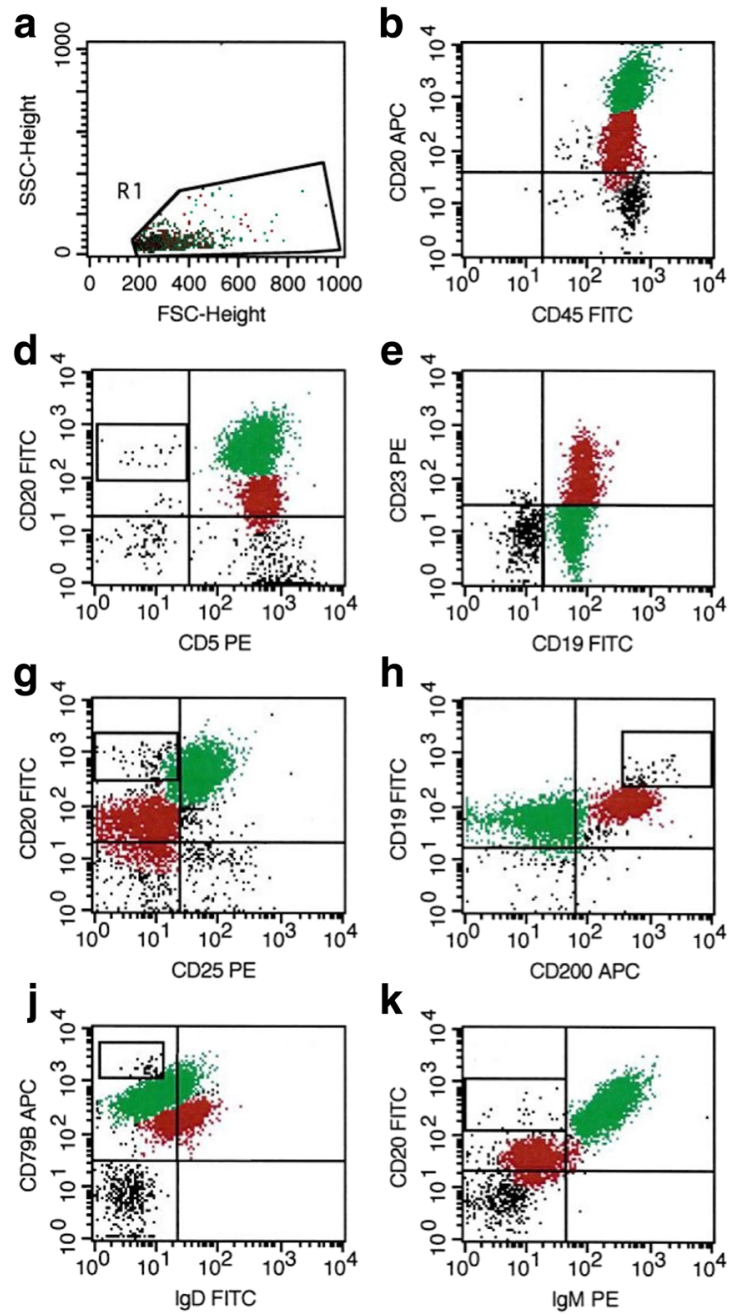
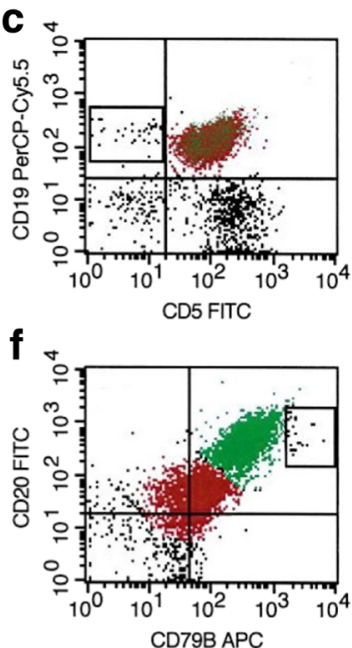

i
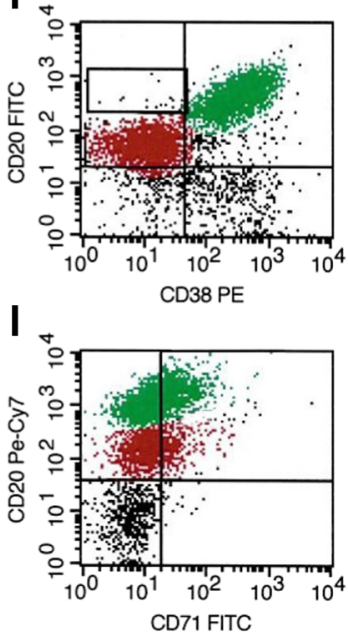

indicated a decreasing level of MRD. At the end of the treatment, both qPCR and FCM showed the lowest levels of MRD indicating a good response to the treatment. Unfortunately, before the next planned MRD assessment, the patient was diagnosed with the progression of SLL. The SLL progression was confirmed by qPCR. No samples were taken for FCM at that moment. Due to the very rapid and unexpected progression of the less aggressive SLL subclone (Fig. 4, bottom, left), the previously planned early detection of the molecular progression as well as the prompt start of SLL treatment could not be initiated.

\section{Materials and methods}

\section{Histology and immunochemistry}

After fixation in neutral buffered formalin, the lymph node was embedded in paraffin and sectioned following routine methods. The sections were stained with hematoxylin and eosin (HE). Immunohistochemical staining with anti-CD20,
CD23, cyclin D1, SOX11, and Ki-67 antibodies was performed according to a standardized automated protocol.

\section{Flow cytometry}

Immunophenotype was also determined by FCM of cellular suspension obtained by FNAB, PB, and BM. All samples were prepared by the standard technique and were stained for 4-5 color immunophenotyping with a broad panel of monoclonal antibodies conjugated with one of the following fluorochromes: FITC/PE/RPE/PerCP/PerCP-Cy-5.5/PE-Cy7/ APC (Becton Dickinson, BD) as previously described [11]. Data was gathered from the FACSCanto II flow cytometer and processed using the BD CellQuest Pro software (BD).

\section{Classical cytogenetics and FISH}

LN cells obtained by FNAB and BM cells were fixed directly and cultured for $24 \mathrm{~h}$ without mitogen or for $72 \mathrm{~h}$ with DSP-30 ( $2 \mu \mathrm{M}$; TIB MolBiol, Berlin, Germany) together with IL-2 (200 U/ml; R\&D Systems, Minneapolis, MN, USA). These 

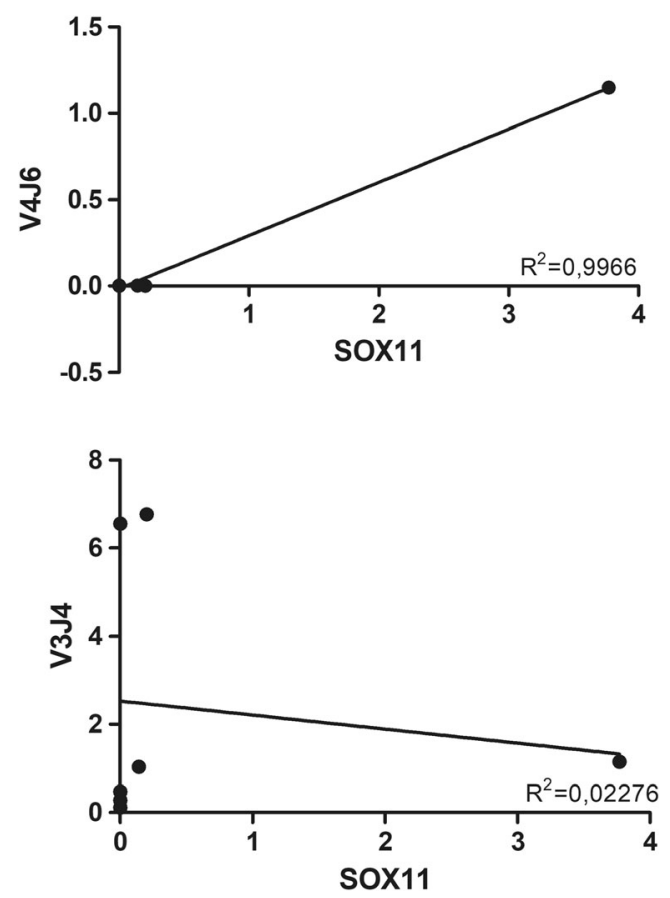

Fig. 3 The regression analysis graphs for V3J4 and V4J6 rearrangement with SOX11 expression. For V4J6 and SOX11 $\left(R^{2=} \sim 1 ; p<0,05\right)$, changes in SOX11 expression explain changes in V4J6. For V3J4 $\left(R^{2}<\right.$ 0,$05 ; p>0,05$ ) - changes in SOX11 expression are not associated with changes in V3J4 rearrangement

cultured cells were used for CC and FISH. The G-banding was performed by standard methods. FISH was performed with the use of probes: D13S319, TP53, ATM, CEP12, and CCND1/IGH (Vysis Abbott Molecular, Downers, Grove, IL, USA).

\section{Molecular studies}

$I G H$ rearrangement and $S O X 11$ expression analysis were performed for PB and BM samples. White blood cells were isolated using a standard gradient separation protocol. DNA and RNA were isolated according to the manufacturer's protocols using the Sherlock AX isolation kit (A\&A Biotechnology) and the GeneMATRIX Universal Purification Kit (EURx), respectively. For the DNA studies, a standard qPCR reaction was performed according to the BIOMED-2 Concerted Action BMH4-CT98-3936 protocol [7]. The expression studies were performed in a two-step RT-qPCR reaction according to a previously published protocol $[16,17]$. Sensitivity of molecular markers was defined by the lowest possible dilution detected by qPCR. The quantitative measurement range was set up as the lowest detected dilution which had a $\mathrm{Ct}$ lower than that of the negative controls background by three cycles. MRD level was calculated to allow comparisons between molecular markers to be made. Results from diagnostic samples were set to a $1(100 \%)$ MRD level, while MRD level in follow-up samples was calculated as derived proportions.

\section{Discussion}

The correct diagnosis of composite MCL and SLL is very important and challenging. Distinction between the components of CL is crucial, since MCL, which is the more aggressive disease, should be treated first. This type of CL is very rare, which only few cases having been reported $[1,15,18]$. Some authors suggest that biopsies containing CLL-type cells but lacking proliferation centres and with non-enlarged or only slightly enlarged lymph nodes on imaging represent a very indolent disease that may be considered a tissue equivalent of monoclonal B cell lymphocytosis rather than overt SLL [12]. However, in our CL case, we received 4 enlarged lymph nodes, each of them approximately $2 \mathrm{~cm}$ long. In addition, FNAB/FCM was performed from an enlarged 3-cm axillary lymph node. This type of CL may be underdiagnosed as the two neoplasms under consideration have many common features. Both malignancies are $\mathrm{CD} 20 / \mathrm{CD} 19 / \mathrm{CD} 5$ positive small $\mathrm{B}$ cell neoplasms, and the correct distinction between MCL and SLL by FCM is possible using a broad panel of antibodies as previously described $[1,2,11]$. Additionally, during the treatment, some immunophenotypical markers (e.g., CD20) might be missing on tumor cells making correct detection of the residual disease by FCM difficult. Recently published work recommends the use of two standard panels for FCM and IHC to distinguish MCL from SLL in CL [1]. As we have shown here and previously $[2,11]$, FNAB is a highly efficient method of obtaining tumor cells for FCM, CC, FISH, and molecular studies in MCL and SLL.

The importance of MRD assessment in therapy decision has been increasing over the last years [6]. Molecular PCRbased MRD estimation presents higher sensitivity $\left(10^{-5}\right)$ comparing to standard FCM $\left(10^{-3}\right)$ and is highly standardized [6].

The methods of distinguishing both malignancies are normalized for diagnostic purposes. However, MRD monitoring of MCL and SLL separately during treatment is still not commonly used. MRD monitoring enables to detect molecular relapse and improve treatment decision [19], and PCR-based methods are " the gold standard" for measuring MRD. The most widely used molecular marker for analyzing MRD in mature B cell lymphomas is $I G H$ rearrangement [7]. This method is highly specific for the identification of a certain fraction of mature B cells (including B-NHLs). However, it is impossible to distinguish which $I G H$ rearrangements are specific to SLL and MCL in CL without a separate molecular analysis of each of the $\mathrm{CL}$ components, which is not possible in PB. Our analysis of $I G H$ rearrangement revealed two clones of malignant mature B cells: with V4J6 rearrangement and with V3J4 rearrangement. The V4 recombinants are found in $29 \%$ of MCL and $10 \%$ of SLL, while V3 recombinants are found in $46 \%$ of MCL and $30 \%$ of SLL [7]. As both rearrangements are found in MCL and SLL, for proper distinction of these clones, we used an additional molecular 

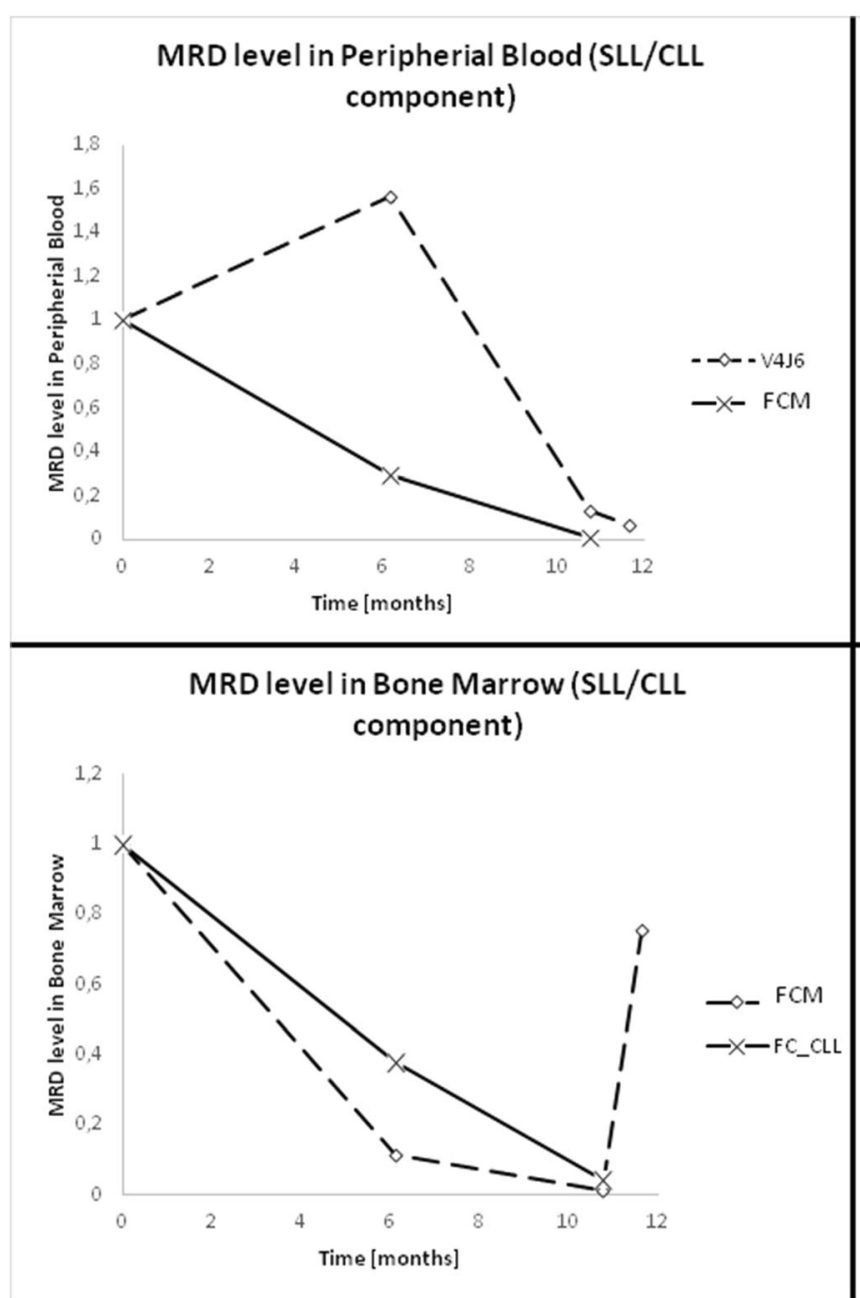

Fig. 4 Minimal residual disease level SLL and MCL components estimated by different molecular markers in peripheral blood and bone marrow. As minimal residual disease (MRD) estimated by molecular markers and flow cytometry had different range of values, the resulting

marker highly specific for MCL. SOX11 expression is found in over $90 \%$ of MCL cases $[3,20]$ and can be used as an MRD monitoring marker, as we previously reported [16].

In this report, we have shown that qPCR analysis may help to differentiate the CL components, especially when supported by SOX11 gene expression analysis. Applying the SOX11 marker allowed us to distinguish the clones and further monitor the disease by standard molecular markers. To our best knowledge, this is the first example of the use of mMRD estimation for the differentiation of the CL components. However, it should be noted that challenges may also occur using molecular methods. In some cases, the proper distinction between clones by IGH rearrangements is impossible due to the polyclonal background, close locations of loci, or inability to detect the $\mathrm{t}(11 ; 14)(\mathrm{q} 13 ; \mathrm{q} 32)$ translocation specific for MCL using PCR, as it was in this case. While this translocation is detectable in 80 to $90 \%$ of MCL by FISH, PCR is able to detect it in only 25 to $40 \%$ [7]. Therefore, it seems
MRD level in Peripherial Blood (MCL component)

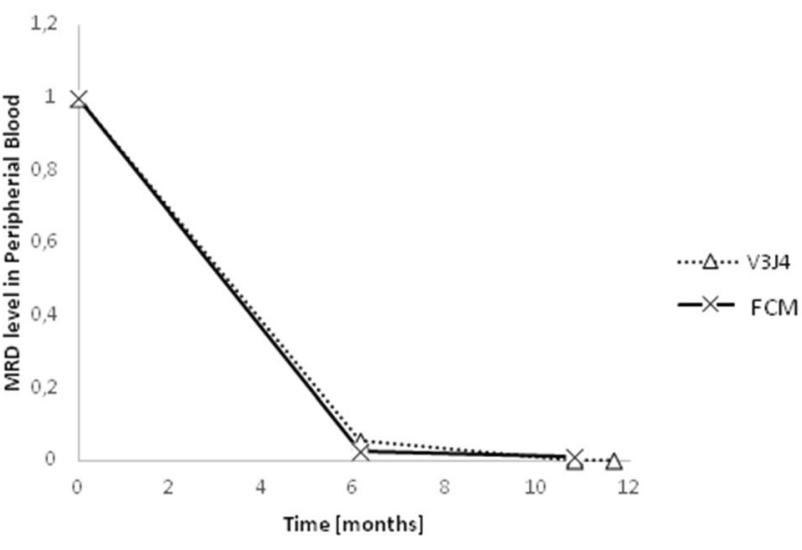

MRD level in Bone Marrow (MCL component)

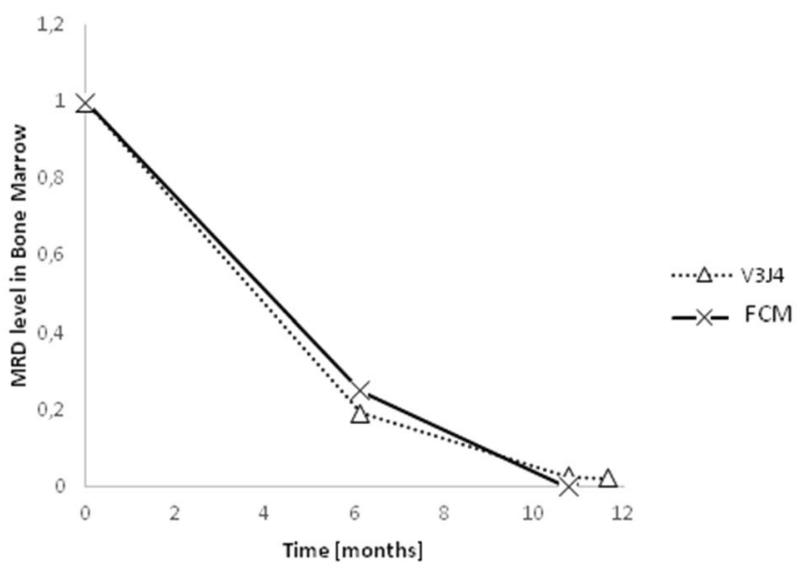

MRD levels were averaged: at the time of diagnosis, the MRD level was set as 1, and the follow-up values were calculated as a percentage of diagnostic sample

crucial to support the classical diagnostic methods by applying genetic and molecular methods in the process of diagnosis and monitoring. As we have shown, applying additional molecular marker SOX11 may allow proper differentiation of the clones in CL.

\section{Compliance with ethical standards}

Conflict of interest The authors declare that they have no conflicts of interest.

\section{References}

1. Sun Y, Wang SA, Sun T (2018) Composite mantle cell lymphoma and chronic lymphocytic leukemia/small lymphocytic lymphoma. Cytometry B Clin Cytom 94(1):148-150. https://doi.org/10.1002/ cyto.b.21512 
2. Woroniecka R, Pienkowska-Grela B, Grygalewicz B, Rygier J, Witkowska A, Rymkiewicz G et al (2002) Significance of chromosomal markers in the diagnosis of mantle cell lymphoma (MCL). J Appl Genet 43(4):545-553

3. Mozos A, Royo C, Hartmann E, De Jong D, Baro C, Valera A et al (2009) SOX11 expression is highly specific for mantle cell lymphoma and identifies the cyclin D1-negative subtype. Haematologica. 94(11):1555-1562. https://doi.org/10.3324/ haematol.2009.010264

4. Dreyling M, Campo E, Hermine O, Jerkeman M, Le Gouill S, Rule $S$ et al (2017) Newly diagnosed and relapsed mantle cell lymphoma: ESMO Clinical Practice Guidelines for diagnosis, treatment and follow-up. Ann Oncol 28(Suppl 4):iv62-iv71. https://doi.org/ 10.1093/annonc/mdx223

5. Pott C, Hoster E, Delfau-Larue MH, Beldjord K, Bottcher S, Asnafi $\mathrm{V}$ et al (2010) Molecular remission is an independent predictor of clinical outcome in patients with mantle cell lymphoma after combined immunochemotherapy: a European MCL intergroup study. Blood. 115(16):3215-3223. https://doi.org/10.1182/blood-200906-230250

6. Pott C (2011) Minimal residual disease detection in mantle cell lymphoma: technical aspects and clinical relevance. Semin Hematol 48(3):172-184. https://doi.org/10.1053/j.seminhematol. 2011.05.002

7. van Dongen JJ, Langerak AW, Bruggemann M, Evans PA, Hummel M, Lavender FL et al (2003) Design and standardization of PCR primers and protocols for detection of clonal immunoglobulin and T-cell receptor gene recombinations in suspect lymphoproliferations: report of the BIOMED-2 Concerted Action BMH4-CT98-3936. Leukemia. 17(12):2257-2317. https://doi.org/ 10.1038/sj.leu.2403202

8. Swerdlow SHBF, Isaacson PI et al (2001) Mantle cell lymphoma. In: Jaffe ES, Harris NL, Stein H, Vardiman JW (eds) Pathology and genetics of tumours of haematopoietic and lymphoid tissues. IARC Press, Lyon

9. Szumera-Cieckiewicz A, Galazka K, Szpor J, Rymkiewicz G, Jesionek-Kupnicka D, Gruchala A et al (2014) Distribution of lymphomas in Poland according to World Health Organization classification: analysis of 11718 cases from National Histopathological Lymphoma Register project - the Polish Lymphoma Research Group study. Int J Clin Exp Pathol 7(6):3280-3286

10. Zamo A, Zanotti R, Lestani M, Ott G, Katzenberger T, Scarpa A et al (2006) Molecular characterization of composite mantle cell and follicular lymphoma. Virchows Arch 448(5):639-643. https:// doi.org/10.1007/s00428-006-0158-9

11. Woroniecka R, Rymkiewicz G, Grygalewicz B, Blachnio K, Rygier J, Jarmuz-Szymczak M et al (2015) Cytogenetic and flow cytometry evaluation of Richter syndrome reveals MYC, CDKN2A, IGH alterations with loss of CD52, CD62L and increase of CD71 antigen expression as the most frequent recurrent abnormalities. Am J Clin Pathol 143(1):25-35. https://doi.org/10.1309/ AJCPATRQWANW2O3N
12. Gibson SE, Swerdlow SH, Ferry JA, Surti U, Dal Cin P, Harris NL, Hasserjian RP (2011) Reassessment of small lymphocytic lympho$\mathrm{ma}$ in the era of monoclonal B-cell lymphocytosis. Haematologica. 96(8):1144-1152. https://doi.org/10.3324/haematol.2011.042333

13. Eichhorst B, Robak T, Montserrat E, Ghia P, Hillmen P, Hallek M, Buske C, ESMO Guidelines Committee (2015) Chronic lymphocytic leukemia: ESMO Clinical Practice Guidelines for diagnosis, treatment and follow-up. Ann Oncol 26(Suppl 5):v78-v84. https:// doi.org/10.1093/annonc/mdv303

14. Pott C, Macintyre E, Delfau-Larue M-H, Ribrag V, Unterhalt M, Kneba M et al (2014) MRD eradication should be the therapeutic goal in mantle cell lymphoma and may enable tailored treatment approaches: results of the intergroup trials of the European MCL Network. Blood 124(21):147. https://doi.org/10.1182/blood.V124. 21.147.147

15. Hoeller S, Zhou Y, Kanagal-Shamanna R, Xu-Monette ZY, Hoehn D, Bihl M, Swerdlow SH, Rosenwald A, Ott G, Said J, Dunphy CH, Bueso-Ramos CE, Lin P, Wang M, Miranda RN, Tzankov A, Medeiros LJ, Young KH (2013) Composite mantle cell lymphoma and chronic lymphocytic leukemia/small lymphocytic lymphoma: a clinicopathologic and molecular study. Hum Pathol 44(1):110-121. https://doi.org/10.1016/j.humpath.2012.04.022

16. Szostakowska M, Szymczyk M, Badowska K, Tudek B, Fabisiewicz A (2018) SOX11 expression as a MRD molecular marker for MCL in comparison with $\mathrm{t}(11 ; 14)$ and IGH rearrangement. Med Oncol 35(4):49. https://doi.org/10.1007/s12032-0181111-x

17. Hamborg KH, Bentzen HH, Grubach L, Hokland P, Nyvold CG (2012) A highly sensitive and specific qPCR assay for quantification of the biomarker SOX11 in mantle cell lymphoma. Eur J Haematol 89(5):385-394. https://doi.org/10.1111/j.1600-0609. 2012.01837.x

18. Papathomas TG, Venizelos I, Dunphy CH, Said JW, Wang ML, Campo E, Swerdlow SH, Chan JC, Bueso-Ramos CE, Weisenburger DD, Medeiros LJ, Young KH (2012) Mantle cell lymphoma as a component of composite lymphoma: clinicopathologic parameters and biologic implications. Hum Pathol 43(4):467480. https://doi.org/10.1016/j.humpath.2011.08.024

19. Luskin MR, Stone RM (2017) Can minimal residual disease determination in acute myeloid leukemia be used in clinical practice? J Oncol Pract 13(8):471-480. https://doi.org/10.1200/JOP.2017. 021675

20. Ibrahim F, Al Sabbagh A, Amer A, Soliman DS, Al SH (2020) Composite chronic lymphocytic leukemia/small lymphocytic lymphoma and mantle cell lymphoma; small cell variant: a real diagnostic challenge. Case presentation and review of literature. Am J Case Rep 21:e921131. https://doi.org/10.12659/AJCR.921131

Publisher's note Springer Nature remains neutral with regard to jurisdictional claims in published maps and institutional affiliations. 